\title{
Rationalizing permeation of polar antibiotics into Gram-negative bacteria
}

\author{
Mariano Andrea Scorciapino, ${ }^{\dagger}$ Silvia Acosta-Gutierrez, ${ }^{\ddagger}$ Dehbia Benkerrou, ${ }^{\ddagger}$ Tommaso \\ D’Agostino, ${ }^{\ddagger}$ Giuliano Malloci, ${ }^{\ddagger}$ Susruta Samanta, ${ }^{\ddagger}$ Igor Bodrenko, ${ }^{\ddagger}$ Matteo Ceccarelli ${ }^{\ddagger}, *$
}

${ }^{\dagger}$ Department of Biomedical Sciences, Biochemistry Unit, and ${ }^{\ddagger}$ Department of Physics, University of Cagliari, Cittadella Universitaria di Monserrato, S.P.8 km 0,700 - 09042 Monserrato (CA), Italy

* To whom correspondence should be addressed

\begin{abstract}
The increasing level of antibiotic resistance in Gram-negative bacteria, together with the lack of new potential drug scaffolds in the pipeline, make the problem of infectious diseases a global challenge for modern medicine. The main reason why Gram-negative bacteria are particularly challenging is the presence of an outer cell-protecting membrane, which is not present in Grampositive species. Such asymmetric bilayer is a highly effective barrier for polar molecules. Several protein systems are expressed in the outer membrane to control the internal concentration of both nutrients and noxious species, in particular, (i) water-filled channels that modulate the permeation of polar molecules and ions according to concentration gradients, and (ii) efflux pumps to actively expel toxic compounds. Thus, besides expressing specific enzymes for drugs degradation, Gramnegative bacteria can also resist by modulating the influx and efflux of antibiotics, keeping the internal concentration low. However, there are no direct and robust experimental methods capable of measuring permeability of small molecules, thus, severely limiting our knowledge of the molecular mechanisms that ultimately control the permeation of antibiotics through the outer membrane. This is the innovation gap to be filled for Gram-negative bacteria.

This review is focused on the permeation of small molecules through porins, considered the main path for the entry of polar antibiotics into Gram-negative bacteria. A fundamental understanding on how these proteins are able to filter small molecules is a prerequisite to design/optimize antibacterials with improved permeation. The level of sophistication of modern molecular modeling algorithms and the advances in new computer hardware, has made the simulation of such complex processes possible at the molecular level. In this work we aim to share our experience and perspectives in the context of a multidisciplinary extended collaboration within the Translocation consortium. The synergistic combination of structural data, in vitro assays and computer simulations have proven to give new insights towards the identification and description of physico-
\end{abstract}


chemical properties modulating permeation. Once similar general rules will be identified, we believe that the use of virtual screening techniques will be very helpful to search for new molecular scaffolds with enhanced permeation and that molecular modeling will be of fundamental assistance to the optimization stage.

\section{A global challenge for modern medicine}

It was November the $11^{\text {th }}, 1945$. During his Nobel Lecture on penicillin, Sir Alexander Fleming said: "It is not difficult to make microbes resistant to penicillin in the laboratory by exposing them to concentrations not sufficient to kill them, and the same thing has occasionally happened in the body." Although, for almost the entire $20^{\text {th }}$ century, antibiotics have been regarded as magic bullets to cure all infections, regardless their use was really appropriate. Almost all antibacterial drugs developed so far, have been followed by detection of resistance.[1] This is the condition acquired by the pathogen, resulting in reduced susceptibility to the action of the drug. Resistance is a natural process related to bacteria evolution, which is accelerated by the pressure exerted by the use of antibiotics in human and human-related activities, as predicted by Fleming. Since bacteria evolve under antibiotic pressure, we will always need new antibiotics to fight bacteria. However, today, our inability to come up with new effective molecules (Figure 1) has turn out simple treatable infections into deadly ones, representing a serious challenge for modern medicine worldwide.
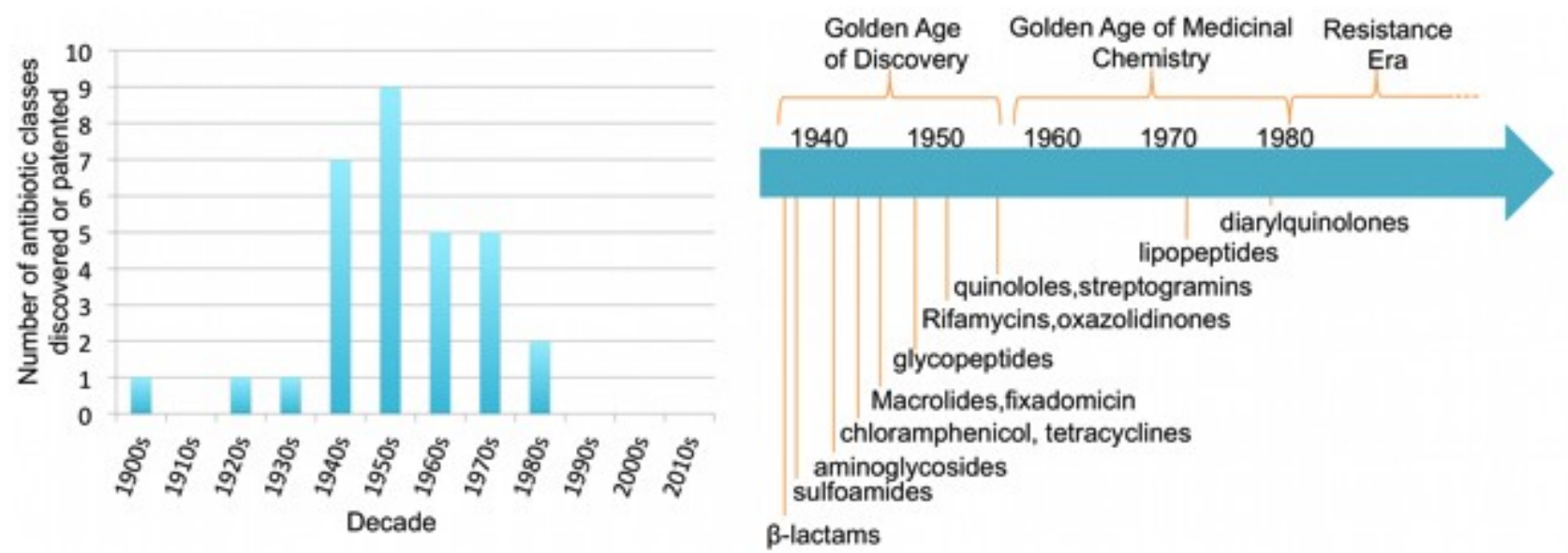

Figure 1. On the left, the decrease in discovery of new antibiotic classes. On the right, antibiotic classes discovery timeline. Source: readapted from Lynn L. Silver, “Challenges of Antibacterial Discovery”.[2]

There are many fundamental gaps in our knowledge about antibiotics, such as the mode of action of many of them, the actual penetration pathway into the pathogen and the mechanisms adopted by the pathogen to actively pump them out or, in general, to develop resistance. Libraries of compounds used within high-throughput screening protocols to identify novel drug scaffolds are now recognized to be biased. This is due to the selection rules applied to the chemical-physical 
properties of the compounds included in such libraries, which are often blindly directed towards oral and/or injectable administration.[3,4] These rules do not necessarily match with other fundamental requirements for a good antibiotic.[4] Conversely, high-throughput screening would be definitely improved by taking into account "rules for good antibiotics”, but these general rules still have to be discovered. This is the main innovation-gap that has to be filled to renew the screening platforms, together with leaving the seek for a novel broad spectrum class of antibiotics, but focusing on case-specific goals.[3,4]

\section{Gram-negative bacteria are particularly challenging}

In the case of Gram-negative bacteria, the problem of resistance is particularly urgent since the pipeline, as reported by The PEW Charitable Trusts Foundation on May 2016, is virtually empty of new scaffolds.[5] Note that we do not want to underrate the great concern of Gram-positive bacteria infections. However, as the World Health Organization has pointed out,[1] the situation is particularly critical for different Gram-negative species. Infections from methicillin-resistant Staphylococcus aureus (MRSA, Gram+ strains resistant to methicillin) strains, for instance, are very difficult to treat but still affordable with glycopeptides like vancomycin and teicoplanin (which are not active against Gram- strains, on the other hand). Even against vancomycin-resistant strains, VRSA, daptomycin and linezolid are available.[1,6] Conversely, multi-drug resistant Gramnegative species such as Acinetobacter baumannii, Enterobacter spp., Enterococcus faecium, Klebsiella pneumoniae and Pseudomonas aeruginosa are responsible for many serious infections in hospitals, where cases of resistance to all the available drugs have already been reported, including the highly toxic colistin, which is usually considered as the last resource.[7]

Both Gram-positive and -negative bacteria have an inner plasma-membrane protecting their interior, but the latter are characterized by a second protective membrane, the outer membrane (OM; Figure 2). The presence of the OM provides Gram-negative bacteria with a very effective intrinsic resistance mechanism, with its permeability properties playing an essential role in their susceptibility to antibiotics.[8,9] OM works in concert with a variety of membrane proteins and protein complexes to keep the entry of needed chemical species as well as toxic compounds under strict control.[10,11] Small hydrophilic antibacterials make use of pore-forming proteins to access the periplasmic space, whereas hydrophobic drugs diffuse across the lipid bilayer.[8] The observation of drug resistance in a large number of species characterized by modifications in the protein or lipid OM composition, bolsters the importance of the OM in antibiotic sensitivity.[9,12] The passive resistance mechanism offered by the presence of the OM is thus additional to the so- 
called acquired and adaptive resistance, which are basically due to the incorporation of new genetic material or a mutation, ultimately resulting in modified proteins and proteins expression levels.[9]

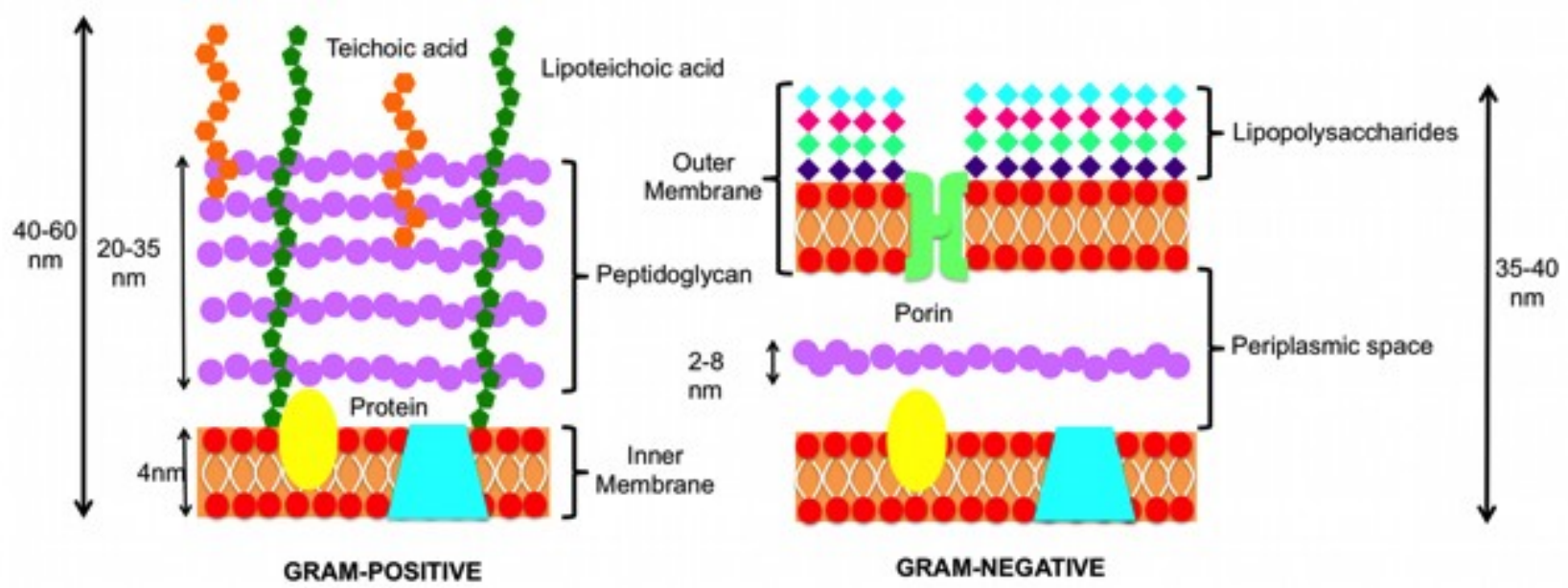

Figure 2. Schematic representation of Gram-positive and Gram-negative cell envelop.

$\mathrm{OM}$ is absolutely peculiar, both in terms of composition and permeability. The presence of the OM, for instance, makes Gram-negative bacteria highly resistant to lysozyme and provides the enteric species with resistance to the detergent action of bile salts and digestive enzymes in the intestinal tracts of mammals.[10] The OM is highly asymmetric in terms of lipid composition, with the inner leaflet composed by phospholipids, while the outer leaflet is formed by lipopolysaccharides (LPS), whose composition and density is highly variable from species to species.[13] While permeability of membrane inner core to small hydrophobic molecules is known,[14] Gram-negative bacteria are usually not susceptible to hydrophobic antibiotics like actinomycin D, macrolides or novobiocin. [10]

Thus, OM is a very efficient barrier for antibiotics. Generally speaking, by slowing the permeation rate into the periplasmic space, Gram-negative bacteria have to neutralize the threat of orders of magnitude less concentrated drug, when compared to the extracellular concentration.

\section{Porins are the main gates for polar compounds}

Porins are typically arranged in the OM either as homotrimers or monomers, with a basic $\beta$-barrel folding, which forms a transmembrane water-filled pore (Figure 3). Porins are needed to reduce OM impermeability to hydrophilic compounds, and are recognized as the main route for the entry of antibiotics with a polar character, such as many beta-lactams and some fluoroquinolones.[11]. 


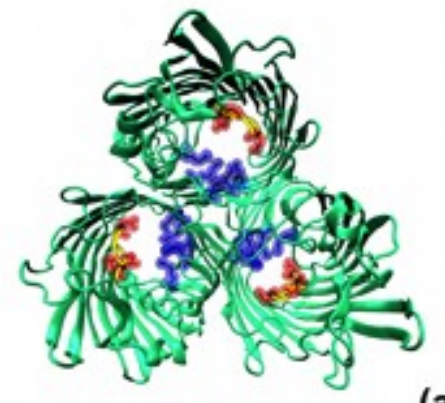

(a)

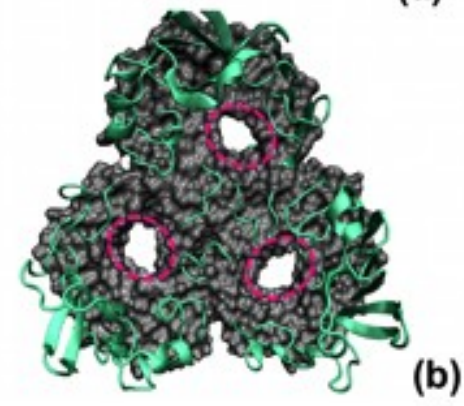

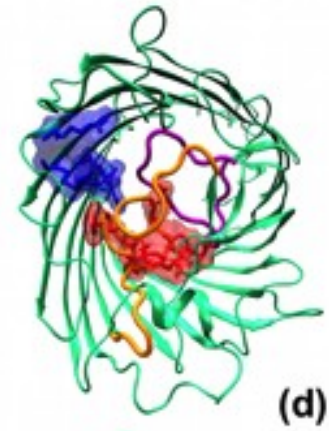

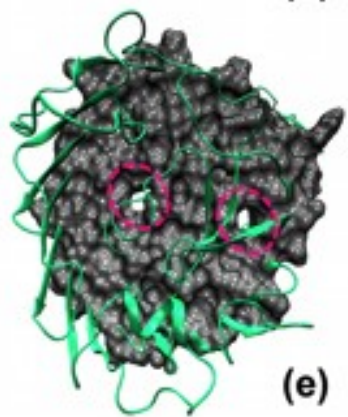

Figure 3. Cartoon representation of a general porin, OmpF from E. coli, and a specific channel, OprD from $P$. aeruginosa. (a) Top view of the trimeric OmpF, where loop L3 has been highlighted in yellow. The oppositely charged residues mostly responsible for the trasverse electric field in the constriction region have been also highlighted in red (negative) and blue (positive). (b) Same as panel a, where atoms Van der Waals surface was added in order to highlight the solvent accessible area inside each monomer. (c) Side view of OmpF, which is shown together with the phospholipid bilayer. (d) Top view of the monomeric OprD, where the loops that folds back into the pore and generate the constriction region are highlighted in purple and orange. The oppositely charged residues that characterized the constriction region have been also highlighted in red (negative) and blue (positive). (e) Same as panel d, where atoms Van der Waals surface was added in order to highlight the solvent accessible area inside the channel.

Some years ago, a combined experimental/computational study was performed by some of the authors on the main entry path of fluoroquinolones. It was shown that a polar character drives the antibiotic through the porin pathway, while more hydrophobic antibiotics prefer to diffuse through the membrane itself.[15,16] Fluoroquinolones, similarly to other antibiotics, can adopt different charged (protonation) states depending on the $\mathrm{pH}$, such that under certain conditions they are able to pass through the membrane or exploit the presence of water-filled pores.[17] The majority of hydrophilic antibiotics do make use of the latter to penetrate and eventually reach their intracellular targets. Thus, it is not surprising to find porins mutation as marker of numerous resistant bacterial strains,[9,18] as we will explain hereinafter.

Usually E. coli is taken as prototypical example for enteric Gram-negative bacteria and, similarly to other members of the Enterobacteriaceae, expresses two principal trimeric porins. In addition to porins, the OM contains also a variety of other proteins with different functions.[11] In the case of E. coli, the major porins are OmpF and OmpC.[19] These are classified as nonspecific general porins, since they allow passage of ions and metabolites up to $600 \mathrm{Da}$ with no clear specificity. Although these two major porins both share a remarkably similar structure and have high sequence similarity, their pore size is different, with OmpC being narrower than OmpF.[19] The observation 
that growth conditions alter the level of expression of these two porins, led to the conclusion that pore size was probably the most important feature that determines channel permeability.[12,19] In particular, an environment characterized by high level of nutrients, such as in the mammal intestine, favors the expression of the narrower OmpC over OmpF.[11] However, numerous computational and experimental evidence are being gathered that point to the role played by internal electrostatics, $[20,21]$ as discussed in details below. It is important to note here, that relative expression of porins is subjected to change with varying the environment composition. This does not apply only to nutrients but also to antibiotics. Indeed, one of the strategies adopted by Gram-negative bacteria to resist antibiotics threat is to reduce the expression of the most permeable porins, while guaranteeing the uptake of nutrients by the overexpression of other channels.[12]

In the case of other species like P. aeruginosa and A. baumanii, OM permeability is lower by a factor $\sim 100$ with respect to that of E. coli.[22,23] Instead of several relatively large and nonspecific general porins, the OM of these bacteria is characterized by the presence of a variety of porins that are specialized for the uptake of the different nutrients needed for growth and survival.[24] Only a few are nonspecific channels (like OprF in P. aeruginosa or CarO in A. baumanii), while the majority are highly substrate-specific and rather small pores, such as the outer membrane carboxylate channel (Occ) family of transporters, also known as OprD family.[25,26] A number of protein channels are also induced under specific growth conditions.[11]

Overall, such a dynamic and tunable OM can offer a variety of resistance mechanisms for Gramnegative bacteria, all of which are basically oriented to antibiotic reduced permeation. These include the reduced expression of wild-type porins, the expression of restricted and more selective channels, expression of mutated porins with altered physico-chemical properties, and the synthesis of channel blockers.[18] In addition to the drug influx problem, drug extrusion by the efflux pumps serves as an important resistance mechanism.[27-29] Efflux pumps are typically characterized by marked poly-substrates specificity. They not only can expel a variety of antibiotic classes, but also act as a primary survival kit for bacteria, helping the acquisition of other resistance mechanisms by lowering intracellular antibiotic concentration. Overexpression of multidrug efflux pumps have been increasingly found to be associated with clinically relevant drug resistance, making Gramnegative bacteria infections a wider challenge.[30] Of course, resistance in Gram-negative bacteria is also obtained by modification of the drug target and by expression of degradation enzymes to reduce intracellular drug availability, similarly to Gram-positive pathogens. However, for Gram negative pathogens the very first step in the action of every antibiotic is the permeation through the outer membrane. Thus, it is clear how a detailed understanding of porins' properties is crucial to 
elucidate their filtering role at a molecular level, in order to make use of this information for optimization of polar drugs.

\section{Porins architecture}

In Enterobacteriaceae, the major porins are general unspecific protein channels, like the prototypical OmpF[31] and OmpC[32] of E. coli. These are homotrimers formed by 18-strand $\beta$-barrels with short turns on the periplasmic side and longer loops on the extracellular one (Figure 3). The contacts between adjacent monomers are stabilized by loop L2, which bends over the barrel wall of the next subunit and forms a salt-bridge with the latter. The size of the lumen is primarily determined by loop L3, which folds into the $\beta$-barrel and reduces the solvent accessible area. Such restricted portion of the channel interior is localized almost halfway through the pore and is referred to as constriction region (CR). Interestingly, while the loop L3 is characterized by several negatively charged residues, it faces the so-called basic ladder, i.e. a series of aligned basic residues located on the opposite side of the barrel wall. An overall hourglass shape usually describes each monomer, where the narrow CR separates the extracellular vestibule and the periplasmic vestibule. The CR presents a remarkable charged residues segregation, which results in a characteristic transversal electrostatic potential gradient, or electric field.[19,33-35]

As already mentioned, the relative expression of $\mathrm{OmpF}$ and $\mathrm{OmpC}$ is highly regulated in E. coli, and it was suggested to be related to the different size of these two pores.[12,36] In particular, the larger OmpF was postulated to permit faster diffusion of nutrients but also noxious compounds than OmpC. Supporting evidences were the environmental factors leading to OmpC higher expression, such as high osmolarity, acidic $\mathrm{pH}$ and high temperature, as like as OmpC was a more restrictive molecular filter than OmpF.[37] Although these two porins share high sequence similarity (77\%) and structure, their filtering properties are significantly different. OmpF has higher conductance than OmpC and a lower voltage threshold for channel closure. In general, they show remarkably different diffusion characteristics towards different solutes as shown by many experimental studies. [10,11,20,36,38-40] However, the molecular reasons for these differences are not well understood, whereas a detailed comprehension of their filtering properties is required to make a significant step ahead against intrinsic antibiotics resistance of Enterobacteriaceae, as well as of other species expressing similar general porins (e.g. Campylobacter jejuni).[41]

For quite a long time, the above mentioned evidences, together with the corresponding lower permeability of OmpC to different classes of antibiotics with respect to OmpF, has lead to the convincing idea that pore size was the primary feature that determined drugs permeability. This was also corroborated by the observation that mutant strains lacking OmpF (and expressing OmpC 
instead) showed decreased antibiotic susceptibility.[12,42] Considerable efforts were spent during the last two decades, and different antibiotic physico-chemical properties have been invoked, such as size, net charge, charge distribution, conformational plasticity and hydrophobicity, to name a few. Nevertheless, no evident strong correlation between the rates of permeation and any of these molecular characteristics has been found so far.[16,20,21,39,42-49] This means no rules are available neither to perform rational molecular screenings nor to design for enhanced permeation. The peculiar hourglass shape of these protein channels clearly suggests a steric barrier, but even taking thermal fluctuations into account, it is not clear how relatively large antibiotics are sometimes found to permeate better than smaller ones. It was quite obvious to look for some protein-substrate interactions that might explain the filtering properties of porins and to focus on the CR (Figure 4).

In the case of bacterial species that mostly express specific porins in their OM, such as $P$. aeruginosa and A. baumannii, finding general rules is even more difficult. Their specific nature suggests case-specific rules for better permeation. Moreover, those channels are usually smaller than general porins, such that getting a molecular picture of how relatively large molecules might be able to pass through is complicated even further. The challenge here is to understand the dynamic of pore expression, in order to focus molecular level investigations. Thus, in this topical review we restrict ourselves on general porins, which have been studied for a longer time and for which more information are available. Possibly, by finding rules for the unspecific porins will shed more light also on the specific ones, suggesting what are the best physico-chemical parameters to look at in more details. Anyway, readers can found interesting examples about specific channel of Gramnegative bacteria in these reports[50,51] and the references quoted therein.

\section{Two barriers one binding site model}

The simplest analytical model to account for the passage of antibiotics through porins is the twobarriers-one-binding-site model (Figure 4), introduced by Benz to describe the passage of maltose, a sugar molecule, through the specific channel LamB.[52] 


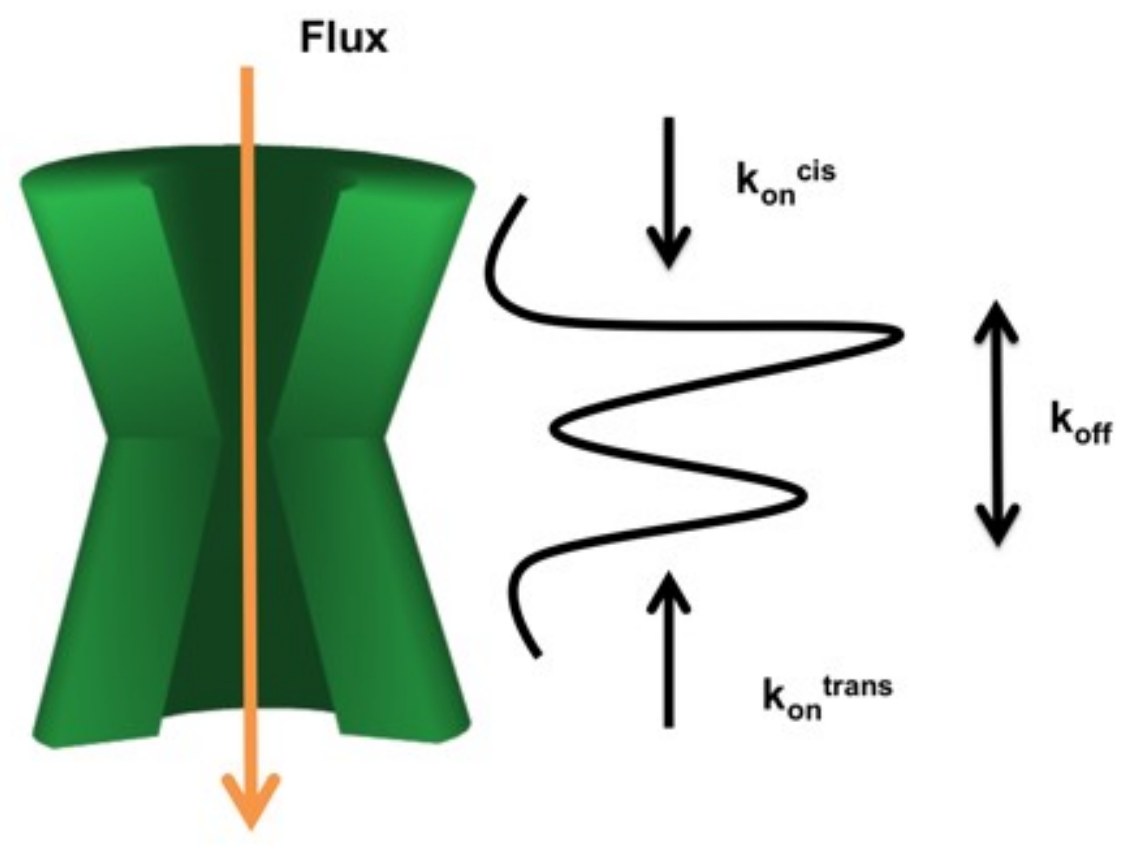

Figure 4. Schematic representation of the "Two-barriers-one-binding-site” model.

In this model, the diffusing molecule is assumed to strongly interact with amino acid residues of the porin's wall, ultimately causing a blockage of the channel that can be measured by electrophysiology techniques as ion current interruptions. The parameters describing the process are the association rate constant, $\mathrm{k}_{\mathrm{on}}$, and the dissociation rate constant $\mathrm{k}_{\text {off }}$, i.e. the inverse of the residence time. The flux of molecules $\mathrm{J}$ can be directly evaluated by a kinetic formulation as a function of concentration $\mathrm{C}:[53] \mathrm{J}=\Delta \mathrm{C} \cdot \mathrm{k}_{\mathrm{on}} / 2$ or $\mathrm{J}=\mathrm{k}_{\mathrm{off}}$ far from saturation and at high concentration, respectively. Such a model requires that the binding site is accessible from both sides of the channel and that the residence time is one hundred microseconds at least, in order to have experimentally detectable events. Molecular dynamics (MD) simulations showed the high complementarity between residues lining the pore and sugar structure, which determined the slow sliding of maltose through LamB and the consequent long time blockage of the channel to ions diffusion.[54]

\section{The electrostatic fingerprint}

The two-barriers-one-binding-site model was developed for specific channels. Aiming at extending the concept to general channels like OmpF and OmpC, in the 2002, Bezrukov and Winterhalther [55] showed that it was actually possible to measure the ion current blockages due to ampicillin diffusing through OmpF. The question was whether a strong interaction between the antibiotic and the channel wall also applied in the case of a general porin. The presence of many charged residues at the CR complements the zwitterionic nature of ampicillin and, indeed, experimental evidence points to local interactions inside the pore. These interactions are most likely of electrostatic nature, 
on the basis of the response of electrophysiology experiments to electrolyte concentration and $\mathrm{pH}$. $[16,21,40,48,55,56]$ When electrolytes concentration decreases, electrostatic shielding inside the pore is reduced and this is typically accompanied by a reduction in the $\mathrm{k}_{\mathrm{on}}$, i.e. a reduced "flux" on the basis of the two-barriers-one-binding-site model.[52] The effect of electrolytes concentration on blockage time $\left(\mathrm{k}_{\mathrm{off}}\right)$ is typically rather low, indicating that it is mostly determined by local interactions. Variation of $\mathrm{pH}$ changes the protonation state (thus the net charge) of both the solute and the porin's residues and, indeed, this usually have a significant impact on electrophysiological measurements.[55]

Starting from the experimental work of Bezrukov and coworkers, [55] we identified the breakage of H-bonds and salt bridges formed by the antibiotic in the CR as the rate-limiting step of translocation. Thus, together with the obvious position of the antibiotic along the channel axis, the number of H-bonds (and salt bridges) was chosen as the collective variables to be accelerated by the metadynamics algorithm.[57] This first study showed i) no large conformational changes of the porin upon antibiotic translocation; ii) an energy barrier to escape from CR of about $10-12 \mathrm{kcal} / \mathrm{mol}$, which corresponds to a time in the order of hundreds of microseconds, in agreement with previous experiments; iii) that molecular flexibility is an important parameter and is helpful for translocation; iv) that charged residues that are not located in the CR can be also important to define the actual escape path followed by the drug. The latter was the case of K16 in OmpF, for instance, whose importance was experimentally confirmed by the altered permeability found as a consequence of its mutation.[58,59]

The picture that emerged from these pioneering works was of strong and detailed antibiotic/porin interactions. The stronger the interaction, the higher the flux and, thus, the efficacy of the antibiotic. The plausible explanation for these conclusions was that the chances of antibiotic translocation through the pore should have increased by increasing the probability to enter inside the CR, which was definitely considered the bottleneck of the whole process. The case of the penicillins, namely, ampicillin, amoxicillin and carbenicillin are particularly interesting. On the basis of the simple twobarriers-one-binding-site model, and by starting from the crystallographic structure of OmpF, a specific pose of ampicillin in the CR was put forward to explain the experimental current blockages. [55] In particular, ampicillin was hypothesized to sit transversally in the CR with its electric dipole matching the oppositely charged residues of the OmpF CR, so that i) the molecule completely blocked the protein monomer, and ii) the resulting strong interaction explained a stable complex for hundreds of microseconds. A few months later, Levadny et al arrived at the same conclusion using a different theoretical approach. Also in their investigation a strong interaction between the molecular dipole and the internal electric field of the pore was suggested.[35] 
Further, Ampicillin was compared to the less hydrophobic amoxicillin and to carbenicillin, all sharing comparable size and the latter having -2 net charge at neutral $\mathrm{pH}[60]$. On the experimental side, amoxicillin showed lower residence time (50 $\mu \mathrm{s})$ compared to ampicillin (120 $\mu \mathrm{s})$, whereas no current blockages were observed for carbenicillin.[48]

In the case of ampicillin, metadynamics simulations showed an additional free energy minimum in the region immediately above the $\mathrm{CR}$, where its hydrophobic side chain interacted with the residues Y22, Y32 and Y40. The minimum for amoxicillin was found closest to the CR and separated by a lower energy barrier to hop into the $\mathrm{CR}$, in agreement with the experimental findings. The behavior of carbenicillin was completely different, by virtue of the repulsion with the negatively charged residues of the loop L3 inside the CR. Starting from the extracellular vestibule, a strong binding site was found above the CR, with a conformation of carbenicillin characterized by a parallel orientation to the main axis of the channel (in contrast to the other two penicillins studied). In principle, the translocation event could be faster than the time resolution of the electrophysiology experiments in the case of carbenicillin, or the strong binding forced the molecule in a position inside the lumen such that ion current was not blocked. The work [48] was left with this open question.

\section{Confirmation and failure of the two-barriers-one-binding-site model}

Further investigations were performed and published in the 2010, [47,49] providing confirmation to some aspects of the problem but giving also evidence of some failure of the two-barriers-onebinding-site model, thanks also to the combined use of a second experimental technique, the liposome swelling assay. Briefly, the porin of interest is reconstituted in liposomes with proper lipid composition. If the antibiotic added to the proteo-liposome dispersion is able to cross the porin with a net influx, water molecules will enter as well, by virtue of the osmotic pressure generated. The resulting swelling of the liposomes is usually recorded by optical density measurements and reported as percentage with respect to a well known good permeant, such as arabinose.[49] However, because of resolution limit and the large variance typically observed, the measured swelling rates can only be taken as an index to rank the antibiotics under investigation in terms of their permeation ability. This assay lacks any molecular level information.

Altogether, the mentioned studies[47,49] supported the idea that a balance between the entropic reduction and the enthalpy gain is needed to optimize translocation. The former is due to the antibiotic confinement in the CR, the latter coming from favorable specific interactions. A coherent picture was found, either when the channel or the antibiotic were modified. In particular, a favorable antibiotic pre-organization was proposed to provide significant contribution to overall reduction of the entropic barrier, i.e. the antibiotic acquiring an optimal conformation for the 
binding before entering the CR.[47] In addition, desolvation upon binding of both antibiotic and amino acid residues in the CR was suggested to provide a favorable entropic increase, as also put forward more recently, and in further details, for OmpC by other authors.[43]

From a general point of view, translocation should be favored when as many antibiotic molecules as possible are attracted at the channel's mouth and, then, inside the extracellular vestibule. The resulting increase in antibiotic concentration on the extracelluar side should increase, in turn, translocation probability. Thus, on this basis, the design of new molecules with high affinity for the channel interior was suggested, which was believed to be accomplished by molecules prone to established a high number of local interactions with the porin's residues. While the $\mathrm{k}_{\text {on }}$ strong dependence on long range interactions was confirmed, on the one hand, also residues at the CR appeared to be able to impact the association rate,[61] on the other.

Finally, it appeared clear that no current blockages measured through electrophysiology does not necessarily mean that the antibiotic is not crossing the channel. Translocation events with characteristic residence time below detection limit are still possible. Conversely, detection of current blockages does not necessarily reflect antibiotic translocation, as shown in the case of enrofloxacin.[16] The molecule might fall into a position inside the channel where it is able to block ion current but where it is characterized by a high energy barrier to proceed further through the pore and ultimately translocate on the other side of the membrane.

\section{To bind or not to bind?}

Does a real binding site help the antibiotic translocation? This fundamental question arises because a robust and direct experimental method able to quantify permeability is still lacking, and this is mainly connected with the physical limits in counting molecules going through nanopores.[62] The Schmitz and Schurr model [63] can be used to estimate the rate of molecules arrival at the mouth of the pore, through the equation $C \cdot k=C \cdot 2 \pi \cdot D \cdot R$, where $C$ is the molar concentration, $D$ the diffusion constant (e.g. $10^{-5} \mathrm{~cm}^{2} / \mathrm{s}$ for ampicillin), and $\mathrm{R}$ the capture radius. When $\mathrm{R}$ is considered as low as 1 $\AA$, at $20 \mathrm{mM}$, which is typical in electrophysiology experiments, the rate of arrival at porin's mouth would result in 1 molecule / microsecond. Then, according to the simple two-barriers-one-bindingsite model,[53] and by using ampicillin association rate of $3000 \mathrm{~s}^{-1} \mathrm{M}^{-1}$, [61] a theoretical flux of 1 molecule every $30 \mathrm{~ms}$ is obtained, which means 1 successful translocation every $3 \cdot 10^{4}$ attempts. From this simplistic evaluation it is clear that the porin, despite providing a facilitated passage through the OM for polar antibiotics, when compared to diffusion through the lipid bilayer, [17] still represents an effective barrier of approximately $8 \mathrm{kcal} / \mathrm{mol}$, obtained applying $\Delta \mathrm{G}=-\mathrm{k}_{\mathrm{B}} \mathrm{T} \cdot \ln (\mathrm{P})$. 
Few years ago, we developed a numerical method to evaluate the net flux according to translocation profiles more complex than just a central well sandwiched between two barriers. [64] Our previous metadynamics simulations showed that additional free energy minima, other than the one inside the CR are possible,[15,16,47-49] suggesting that a multi-well and multi-barrier profile actually applies to antibiotics translocation through general porins. Our numerical approach was based on a kinetic Monte Carlo scheme.[64] The numerical experiments predicted that, at concentrations comparable to those found in-vivo, the only really important parameter is the highest barrier inside the channel, which we placed at the CR, where pore size reaches the minimum. However, at low concentration, an affinity site located near the CR was shown to be important to increase the flux. On the other hand, at high concentration, the presence of a reservoir at the mouth of channel, far from the CR, generates saturation.[64] In conclusion, a free-energy profile characterized by a central barrier can provide an electrophysiology signature similar to that obtained for a system with a central binding site.[16] In terms of estimated flux, a pore without binding site in the CR resulted to be as good as a pore with that feature. Actually the absence of a binding site was expected to be even better, because saturation of the channel should be avoided.

Later, Ziervogel and Roux have shown that a binding site exists for three different $\beta$-lactam antibiotics inside OmpF.[45] They solved the x-ray structure of three independent co-complexes with, namely, ampicillin, carbenicillin and ertapenem, all of which having a distinct binding site outside the CR. These findings suggested that the presence and exact localization of the binding site, if any, was molecule dependent. According to their extensive MD simulations, mutation of key residues in the binding site determined a decrease of the translocation barrier, bolstering the idea that a real binding site is not necessary for translocation. However, it has also to be taken into consideration that even a single point mutation might change significantly the pore size. The authors concluded that "it remains to be seen whether charged residues within the narrow constriction zone play the largest role in determining total solute diffusion rates”.[45] It is plausible that too a high affinity for any internal site can actually hinder, more than promoting, translocation in a concentration dependent fashion.[65]

More recently, the idea of strong direct interactions between the drug and the porin's residues has been questioned.[39] The general electrostatics of the lumen, more than its size, has been indicated to be of primary importance in regulating permeability.[20,42] By using an enzymatic assay based upon the velocity of $\beta$-lactam antibiotic degradation in intact cells, Koijima and Nikaido[39] compared ampicillin and benzylpenicillin. They concluded that, although the presence of drug specific binding sites should have been able to produce some effects on drug diffusion rates, such effects were expected to be only subtle, while rates would have been predominantly determined by 
the general physico-chemical properties of the molecule. Definitely, it was suggested that porins like OmpF and OmpC have to be regarded as general porins, through which antibiotics can pass by virtue of a simple diffusion mechanism, without any overemphasized role played by strong interactions with the channel's wall. In addition, in a successive paper,[20] the same authors bolstered this concept. The permeation properties of OmpF and OmpC have been compared with respect to different antibiotics and by taking into account the actual physiological context where the two porins are mainly expressed, which is low and high osmolarity, respectively. Despite crystal structures show a significant difference in the CR size, the authors concluded that this difference is not as important as the internal electrostatics. When OmpF and OmpC were compared in relatively low osmolarity medium, which is the typical situation in most of the in vitro investigations, OmpC was found to be the less permeable, in agreement with previous studies. However, when a higher osmolarity environment was considered, OmpC permeability was found to increase up to the OmpF level. Now, in high osmolarity media like in mammal intestine, where both nutrients and toxic compounds concentration is high, the bacterium still needs to facilitate the uptake of the former, so that OmpC permeability enhancement is certainly compatible with the physiological context. However, the molecular reasons why OmpC, at the same time, seems to be able to hinder the uptake of noxious species remain to be deciphered.

More recently, researchers from Astra Zeneca investigated the permeability of carbapenems through specific channels of P. aeruginosa.[51] Combining simulations and experimental data on a series of meropenem/imipenem derivatives, they showed how binding at the constriction region can indeed affect the biological efficiency of antibiotics. However channels from P. aeruginosa are classified as specific channels, whereas similar evidence of a biological response has not been measured yet for general porins, as far as we are aware. Numerous open questions are still pressing. What is the actual bottleneck to the translocation process? And, where is it exactly? Is it drug dependent? Or, is it mostly dictated by porin's morphology and physico-chemical characteristics? We definitely need investigation tools able to capture these details and translate them into usable general rules to enhance the efficiency of the present drug design approaches.

\section{Molecular simulations as useful tools}

In the presented scenario, we think that MD simulations are particularly suited. Molecular modeling has the potentiality to provide the requested information, working down at the atomic scale. So far, knowledge of the antibiotic translocation problem have pointed essentially to three mechanisms: (i) slow diffusion,[66] (ii) diffusion with molecule binding,[52] and (iii) a mechanism based on pore dehydration induced by the permeating molecule.[43] These three mechanisms are not to be 
intended as mutually exclusive, in our opinion. In order to discriminate among these three mechanisms, to attain a better description of the translocation process, and to come to a comprehensive picture, quantification of the subtle antibiotic/pore/water interactions is required. Thanks to the high resolution in space and time, molecular modeling makes such detailed characterization possible, in terms of hydrogen bonds, hydrophobic contacts and, ultimately, energetics.[62] The complete control over the characteristics of the system allows to understand the impact of pin-point mutations[50,67,68] and the effects due to different domains of the same proteins [69]. The high resolution in time and space allows to distinguish between effects of different nature[70,71] and to overcome the limitations of currently available experimental methods. The rationalization of the permeation process of antibiotics into Gram-negative bacteria via computer simulations requires an accurate and exhaustive description of some key molecular properties for many antibiotics of different classes. Thanks to the ever-increasing computational power available, the use of modeling tools represents the best alternative to obtain homogenously derived physico-chemical descriptors for molecules, and it can provide a useful guide for future experimental work.

In particular, MD simulations based on all-atom empirical force-fields are nowadays routinely carried out in the microsecond time range and beyond, making it possible to reach a very good level of description of the structural and dynamical properties of biological systems, such as membranes, proteins and nucleic acids. However, the parametrization of generic molecules (drugs, dyes, metabolites, ...) often remains a non-trivial task, despite the efforts in developing (semi-)automatic tools.

Recently, in the framework of the TRANSLOCATION consortium,[72] we started to build a database of computed molecular properties derived from both quantum-mechanics and $\mu$-long MD simulations for a sample of 50 different antimicrobial compounds, ranging in size from $\sim 20$ to $\sim 80$ atoms.[60] For each compound, we generated the General Amber Force Field parameters [73] for the major species at physiological $\mathrm{pH}$, and we performed the analysis of different properties of general interest, including number and population of relevant structural clusters, molecular flexibility, hydrophobic and hydrophilic molecular surfaces, as well as the statistics of intra- and inter-molecular H-bonds, hydration shells structure and dynamics, etc..., as extracted from $\mu$ s-long MD simulations in explicit water. The database includes several key molecular parameters, such as energy of the frontier molecular orbitals, normal modes of vibration, rotational constants, atomic partial charges and electric dipole moment, computed by Density Functional Theory. This database is freely accessible on-line at http://www.dsf.unica.it/translocation/db. To the best of our knowledge, our database is the first extensive one that reports structural, physico-chemical, and 
especially dynamical properties, obtained by combining different computational tools for a specific class of molecules, the anti infectives.

As reported above, the barrier a molecule like ampicillin has to overcome to diffuse through a general porin, such as OmpF, can be estimated to be as high as $\sim 8 \mathrm{kcal} / \mathrm{mol}$. This value, when compared to the average kinetic energy, $0.6 \mathrm{kcal} / \mathrm{mol}$ at $300 \mathrm{~K}$, shows that standard MD cannot be applied to explore the conformational space at the molecular level required for a rational drug design. While the limitations of both experimental methods and computational power have been drastically reduced in the last years, there is still a gap between the time scales that are accessible through simulations and the ones characteristic of experimental techniques. The use of enhanced sampling methods has proven to be extremely useful to obtain information on both the dynamics [67-69] and kinetics [71] of the processes discussed here, despite these methodologies are strongly dependent on the choice of what is considered as the main bottleneck of the process. Finally, the results obtained through the use of metadynamics may present some artifacts that are intrinsically generated by the addition of the energy biases, potentially affecting the transition rates between the states of the system.[71] Following the approach of Doerr,[74] our group is currently investigating alternative methods based on multiple replicas, in order to increase the speed of the exploration without the need to add an external potential. This will be also favored by the future exascale architectures, where thousands of cores will be available at once within a massive parallel scheme.

\section{Porin's electrostatic fingerprint by using water as molecular probe}

Recently, we performed a detailed investigation of general porins' internal electrostatics, by comparing the serial mutations observed in successive E. coli resistant clinical strains. In particular, seven strains were isolated during treatment of a patient suffering from Caroli syndrome over two years, including both cephalosporins and carbapenems, but also fluoroquinolones.[75] The isolation of these strains offered the rare opportunity to investigate the evolution of bacteria within the same patient, with progressively greater antibiotic resistance along the series. That first study was complemented, later on, by Lou et al.,[42] with the publication of the high-resolution crystal structures of four OmpC mutants, namely, OmpC20 from the first clinical isolate, OmpC26 with mutation D18E, OmpC28 with D18E and S271F and OmpC33 with D18E, S271F and R124H substitutions. Interestingly, these mutations did not result in significant changes in the pore size, with just small changes in ion conductivity.[42] The availability of these crystal structures allowed us to perform an atom-level computational comparative study of the wild-type OmpF and OmpC, and the two OmpC clinical mutants OmpC20 and OmpC33, the first and the last one, respectively, along the series. 
Both the loop L3 and the basic ladder, i.e. the two main element of the CR, were found to be highly conserved, even among the wild-type OmpF and OmpC, despite their relative expression is known to strongly depend on the environment osmolarity.[12] All the investigated porins had a net negative charge, which increased from OmpF to OmpC and that was even larger in the two OmpC mutants. However, such a difference in net charge was not evenly distributed along the channel. In particular, a residues cluster around OmpF's R167 and R168 was found to be mutated along the series OmpF, OmpC, OmpC20 and OmpC33, such that formal net charge of the cluster was $+1,0$, 3 and -3 , respectively, located at the mouth of the CR.[67]

Because water molecules naturally fill these general porins and due to their relatively large dipole moment in spite of the small size, they can be used as natural probes for channel's internal electric field (EF). Starting from the pioneering work of Karshikoff,[34] several studies were devoted to the characterization of the EF inside general porins [76-78] and, interestingly, a screw-like field was already suggested for OmpF.[77] In our work,[67] a remarkable local rearrangement of water molecules in the direction of the internal EF was identified across the channel, with striking differences among the four investigated porins. The main differences were found in the CR and at the interface between the extracellular vestibule and the CR. Within our computational procedure, the protein channel was divided into adjacent sections of identical thickness (5 $\AA$ ) along the main axis, and the local polarization density vector (the dipole moment per molecule per volume) of water was calculated for each section. Inside all the investigated porins, water order was maximum in the $\mathrm{CR}$ and almost perpendicular to the main axis of the channel, clearly suggesting a strong transversal EF pointing from the positive basic ladder to the negatively charged loop L3. In addition, all of the four porins shared $\sim 90^{\circ}$ azimuth rotation of water polarization density vector upon emerging from the CR to the periplasmic vestibule.

Conversely, as already mentioned, striking serial differences were found at the mouth of CR on the extracellular side. In OmpF, the water polarization vector showed virtually the same direction all the way through the CR, starting from the mouth down to the above mentioned $90^{\circ}$ azimuth rotation on the periplasmic side. Similar profile was also found in OmpC, but the magnitude of water polarization vector was dramatically reduced right at the mouth of CR, which was referred to as the "pre-orientation region". In the same region of OmpC20, the direction of the water polarization was inverted. The same was observed also in OmpC33 with an even higher strength. It was particularly interesting to observe that specific amino acid mutations were introduced in the two resistant mutants to tune the transversal EF right at the entrance of the CR, while the size was almost left unaffected. 
The following picture emerged from our study: while the translocating molecule experiences a dramatic decrease of conformational entropy upon entering the CR, the resulting free energy increase can be compensated by the favorable orientation of its electric dipole with respect to the channel internal EF (Figure 5). In our opinion, these are probably the two most important factors that ultimately control the permeation of antibiotics through general porins. A preorientation region just above the CR, where the dipolar drug is driven to assume an orientation which is already favorable to enter the $\mathrm{CR}$, like in $\mathrm{OmpF}$, was predicted to facilitate the overall translocation process. Thus, conversely, a weak pre-orienting field like in OmpC or, even worse, an electric field pointing in the opposite direction like in the two resistant mutants, is expected to impose an adverse preorientation exactly where steric hindrance to molecular reorientations starts to be severe, i.e. right at the mouth of the CR. [67]
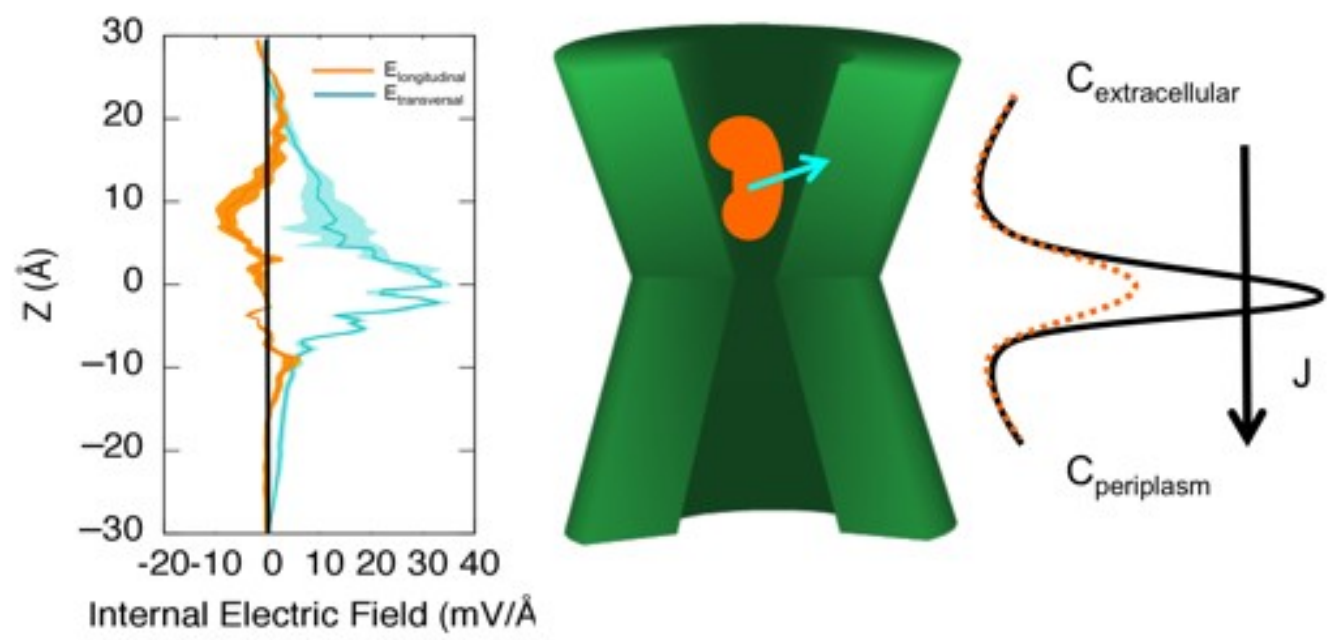

Figure 5. Electric field filtering mechanism. The steric barrier in the constriction region, (black solid line in (c)) is reduced (dashed orange line in (c)) by the interaction of the electric dipole moment of the molecule (b) with the internal electric field of the pore (a).

More recently, we suggested a computational method to quantitatively determine the macroscopic EF inside these nano-sized water-filled channels from all-atom MD simulations.[79] The strategy was not based upon an initial guess of the dielectric function of the inhomogeneous poremembrane-solvent medium, like it is accepted in the more traditional Poisson-Boltzmann approach. Differently, our approach was based upon the direct use of the non-linear dielectric response function of water as it was calculated in a separate all-atom MD simulation (Figure 6).[79] This approach is not only relevant to water-filled channels, but can be extended to calculate the EF along the external surface of proteins (or other biological systems) or in water-filled cavities, the only prerequisite being the presence of enough water molecules to be used as probes. 
Water analysis was performed from MD trajectories extracted for each protein. In the case of the porins under consideration, a cylinder of $80 \AA$ height and $17 \AA$ radius was used.[79] The geometrical center of the distribution of oxygen atoms was calculated every $0.5 \AA$ along the channel axis (z-axis). Then, a spherical water probe with radius of $5 \AA$ was considered for each point obtained along the diffusion path, for each trajectory frame. The polarization density of each sphere of water molecules was calculated and averaged over the whole MD trajectory. Finally, because the protein channels under investigation were trimeric, trajectory averages were also averaged over the three equivalent monomers, which could be considered as statistically independent systems.

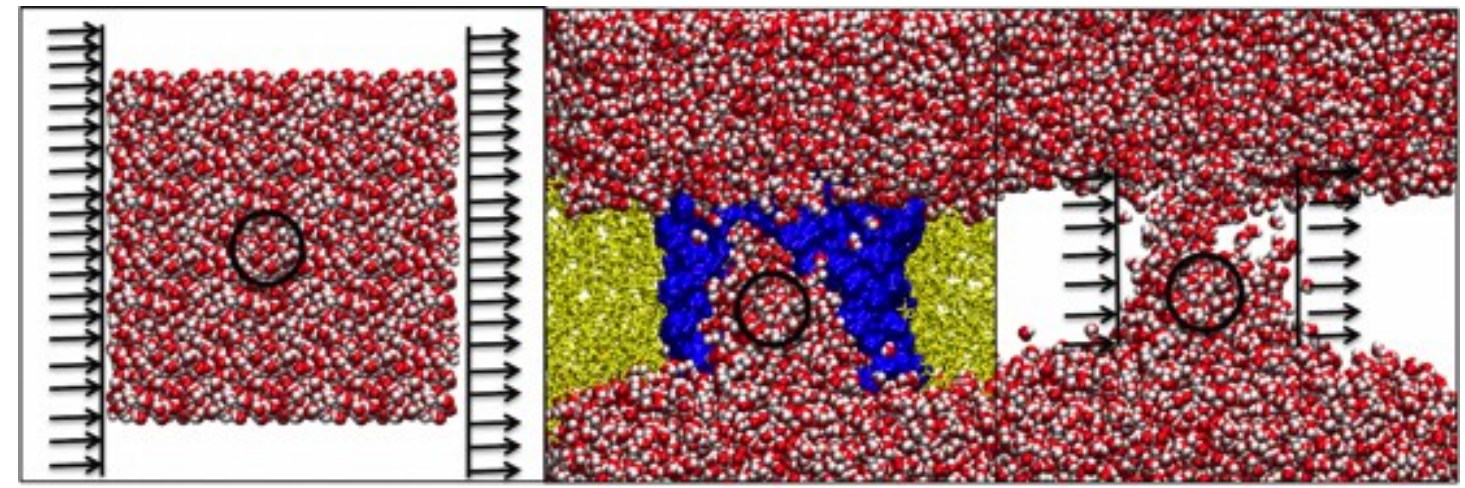

Figure 6. Schematic representation of the approach used to calculate the intrinsic electric field created by the porins through the water polarization. (Reused from ref. [79]) A spherical volume of water molecules shows a polarization that is dependent on the external applied field, as we estimated for bulk water (left panel). A certain degree of polarization was observed for the same spherical volume of water molecules inside the porin (in blue) at different depth (central panel). Thus, on the basis of water molecules polarization, we could estimate the electric field strength generated by porin's internal electrostatics at different depth (right panel).

The presence of a strong transversal component of the EF with the greatest strength in the CR was clearly shown (20-40 mV $\AA^{-1}$ ), while z component was almost negligible (Figure 7). OmpF resulted to be very sensitive to the electrolyte concentration. In particular, the y component of the EF in the CR was dramatically reduced already at $200 \mathrm{mM} \mathrm{KCl}$, down to values comparable to the ones characteristic of OmpC. Also the pre-orientation region was remarkably perturbed, so that the overall profile of the two main porins of E. coli became comparable. Indeed, when the same conditions were applied to OmpC, no remarkable differences in the intrinsic EF were found, up to $400 \mathrm{mM} \mathrm{KCl.[79]}$ 


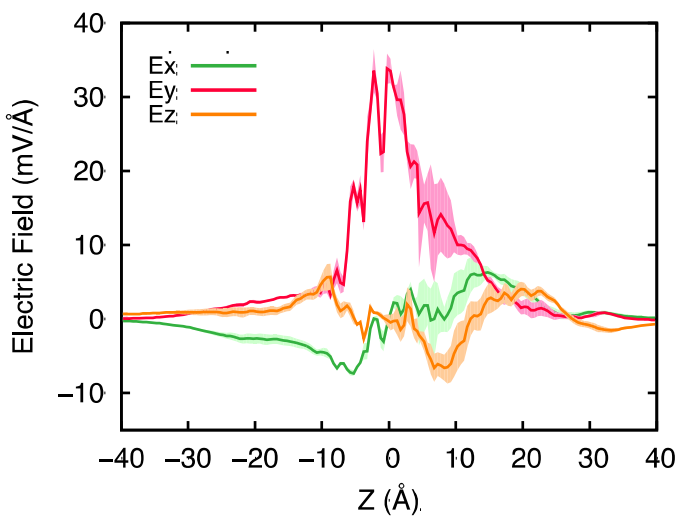

(a)

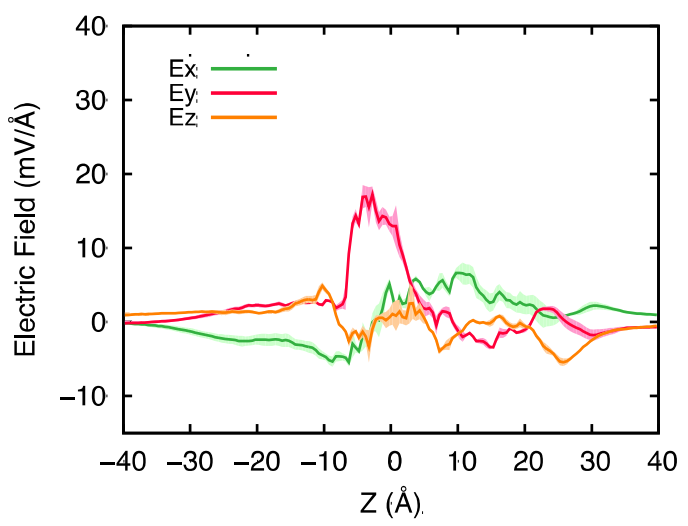

(b)

Figure 7. Internal electric field inside (a) OmpF and (b) OmpC. (Reused from ref. [79])

It is very interesting to note how these results match the above mentioned observations about the relative expression of OmpF and OmpC in different environments and growth conditions.[12,20,39] In an environment characterized by nutrient deficiency, a general channel should guarantee a sufficient influx of essential species. We suggested that an OmpF-like profile of the EF at low osmolarity, with high field strengths both in the CR and pre-orientation region, is helpful for the uptake of dipolar molecules. However, this is the case of most of the commonly employed antibiotics, which are characterized by a significant electric dipole, making bacteria expressing OmpF rather susceptible. Under the same conditions, OmpC expressing bacteria are generally less susceptible, probably because of the lower field strength in the CR and, most importantly, to the negligible pre-orientation field strength.

OmpF and OmpC clearly appeared to be designed by evolution to work under different environmental conditions. The electrostatics of OmpF is profoundly affected by the presence of electrolytes in the medium, thus, it is not surprising to find this porin favorably expressed in low osmolarity media. On the other hand, OmpC is almost insensitive to the ionic strength, preserving its inherent electrostatic profile, and probably correlated higher filtering power, even in the presence of electrolytes at fairly high concentrations. However, for the sake of clarity and in agreement with all of the above quoted references, beside protein's internal EF, it is important to stress that other factors cannot be neglected, such as substrate size and flexibility, the net charge as well as $\mathrm{H}$ bonding propensity, hydrophobicity and desolvation upon binding. Drugs permeability through bacterial general porins is a multivariate process, where substrate specific properties do probably play a role over the channel specific background.

\section{How to exploit the internal electric field to drive drugs through the pore}


Similarly to the water molecules, that tend to align their electric dipole to the porin's internal electrostatics and to follow any variation along the channel axis, the same EF profile inside the channel was suggested to have a macroscopic effect on transit of larger molecules such as polar substrates[80] and polar antibiotics.[67] By taking the opportunity offered by the above mentioned clinical series of OmpC mutants,[42] we investigated the effect of those serial point mutations on antibiotic uptake and the correlation with the predicted internal EF modifications.[81] Liposome swelling assays showed that permeability was lower through OmpC33 than OmpC20 for both imipenem and meropenem, in good agreement with the decreased susceptibility observed in the corresponding clinical strains. Despite electrophysiology does not provide direct measure of translocation, it was important to estimate the relative affinity of the antibiotics for the different porins.[81] A rather good agreement was found with MD simulations. Antibiotic accessibility resulted clearly asymmetric in all the cases but imipenem/OmpC33, with the higher free energy, on average, to enter from the extracellular side than from the periplasmic one. MD simulations clearly showed the adverse orientation adopted by both investigated carbapenems while approaching the CR from the extracellular side. In agreement with our previous investigations on the water molecules,[67,79] this behavior was more pronounced inside OmpC33 than OmpC20.

A very recent systematic comparison of imipenem and meropenem diffusion through OmpF, OmpC, and the two clinical strains mutants, OmpC20 and OmpC33, showed new interesting details. [68] An altered number of hydrogen bonding or charged groups in the CR could not be invoked to explain the differences observed in the translocation free energy of each of the two carbapenems, since no amino acid mutations are present in the CR.[67] In addition, all the four porins were comparable in size,[42] thus pointing to the variations of the EF direction along the diffusion axis of the pore as the main factor ultimately determining translocation performance. The optimal orientation of antibiotic's electric dipole inside the CR is clearly the one directed from the positive basic ladder toward the negative loop L3. In OmpF, this orientation was already adopted before entering the CR, when the antibiotic was still in the pre-orientation zone. Correspondingly, a relatively easy accessibility was observed for the two antibiotics when approaching the CR from the extracellular vestibule.[68] In OmpC, the almost ineffective pre-orientation EF caused the drug to be not properly oriented before entering the CR. Indeed, the free energy barrier for translocation was observed to start before the actual CR. In OmpC20, pre-orientation was strong and pointed $\sim 180^{\circ}$ away from the optimal direction to descend into the CR, such that an inversion of the dipole orientation was needed while the molecule proceeded by less than $2 \mathrm{~nm}$ along the translocation path. Finally, in OmpC33, such adverse pre-orientation was effective up to a very short distance 
from the CR. Correspondingly, the free energy barrier was extremely wide and this reflected by the antibiotic never reaching the proper orientation inside the CR.[68]

In order to effectively compensate the entropic cost due to desolvation and drug confinement inside the CR, the importance of the alignment of the antibiotic's electric dipole to the porin's internal EF emerged from our studies. The EF is often almost perpendicular to the channel axis, so that, by taking the same axis as the $\mathrm{z}$ of our reference coordinate system, xy reorientations are mostly needed by the crossing antibiotic. However, we showed that xy dipole orientation is not sufficient to capture the translocation process. Actually, the magnitude of the dipole xy-projection has to be taken into account. Despite an apparently good xy orientation, the antibiotics is sometimes entrapped in specific configurations that are characterized by a low xy projection, explaining the relatively high energy barrier for translocation in those instances.[68] In this scenario, a closer inspection of the molecular properties of the investigated antibiotics suggested that the molecule with the best permeation properties shall be the one with a certain angle between the main axis of inertia and the electric dipole. In other words, the "best" antibiotic is able to both physically fit the shape of the CR, aligning its long axis with the pore's one, and to align the electric dipole to the pore's EF at the same depth. For instance, we expect that a molecule with the electric dipole directed along its main axis of inertia is not a good permeant, because it would be forced by the porin's EF to adopt a transverse orientation inside the CR, thus, maximizing the steric hindrance. In conclusion, we put forward a new model, "align and translocate”, in contrast to the former "bind and translocate” concept. During translocation the antibiotic must fulfill a set of conditions inside the porin's CR, corresponding to a restricted number of conformations. The electric dipole should match the channel electrostatics and the best conformation should be also compatible with the correct orientation of the H-bonding groups. The molecules with the best permeation performance are predicted be the ones endowed with enough flexibility to change direction of their electric dipole easily, with low-cost dihedral torsions of specific groups, without the need for more “expensive” molecular reorientations. Hopefully, by taking these concepts into consideration will help the design of novel antibiotics. The concepts presented here can contribute to provide the basis for future in-silico screening aimed at identifying molecules with enhanced permeation properties.

\section{Specific porins as evolution of general porins}

While the outlined approach has been assessed by investigating general porins like OmpF and OmpC from E. coli, we are currently applying the same approach to substrate-specific porins of $P$. aeruginosa and A. baumanii.[50,69] The large number of these highly homologous porins makes specificity in substrate transport not yet completely clear. In P. aeruginosa, the Occ family of porins 
has been classified in two broad classes - cation selective OccD, and anion selective OccK subfamilies.[82-84] However, both show the same segregation of charges typically observed also in general channels, with a basic ladder on one side and anionic residues on the other. We believe that the internal EF might play a role in modulating translocation of substrates also through the specific channels.

Differently from general channels, the eyelet region is lined by two long flexible loops that seem to work as a gate, as recently shown for OpdK/OccK1.[85] More interestingly, the dynamics of the two loops can create two alternative paths in OprD/OccD1.[50] Substrates appeared to use a different path depending on their charge distribution and polarity. Charged and polar molecules follow a more polar path, whereas neutral molecules tend to pass through the less polar one. The presence of a negative pocket just above the polar path appeared to hinder the passage of negative substrates through OprD. On the other hand, molecular simulation studies showed the presence of a series of binding sites that apparently facilitate the translocation of positively charged substrates, arginine in particular, through OprD.[86] OprE/OccK8 of this family is speculated to transport double negative cephalosporins.[87] Apart from this, not much information on the molecular details of substrate translocation through the Occ family is available. The importance of specific channels is that, as mentioned earlier in this review, they showed a macroscopic effect on antibiotics transport. Small modifications on the chemistry of imipenem/meropenem provided a different affinity to the OprD and this was enough to create a drastic change in MIC.[51] In particular, it was proved that by increasing the affinity for the target was not sufficient to improve the antibiotic power because of a decreased affinity for OprD. In the future we hope to show the opposite: by increasing the affinity for the pore and, ultimately, the translocation performance, will be sufficient to have an antibiotic with enhanced in vivo activity.

\section{Future perspectives}

The kinetics of an antibiotic molecule that passes through a porin channel must be characterized at different time scales. First, the diffusive reorientation of a free molecule occurs in the 10-100 ps timescale. Then, antibiotic's diffusive redistribution in the vestibule of a porin, in the absence of strong binding sites and significant energy barriers, can take up to 1-10 ns. The residence time due to strong binding of the molecule in the pore is in the micro- and the millisecond range, as it is determined by means of electrophysiology experiments.[88] Finally, the average time between successive translocation events, at the physiologically relevant concentrations of the solute, may be up to seconds. 
Each of the available theoretical and experimental methods for the characterization of the antibiotic translocation process works well in different portions of this almost 9 orders of magnitude wide temporal range. Today, the all-atom MD simulations allow to analyze the molecular processes in the time scale of few microseconds, due to the limitations in the computational resources.[81] The available experimental techniques (e.g. electrophysiology and liposome smelling assay) can provide molecule-pore interaction kinetics in the millisecond time scale.[88] The challenge is to bridge the gap between the two time-scales, in order to achieve a better understanding of the microscopic mechanisms that ultimately regulate antibiotic translocation through bacterial porins.

In the following, we will discuss possible directions to extend the theoretical analysis of the molecular transport kinetics based on the all-atom simulations to the above-microsecond time scale. It is worth to note that the extension of the experimental methods to quantify the sub-microsecond pore-substrate interaction kinetics is also possible.[70]

The enhanced sampling techniques in all-atom simulations (e.g. metadynamics[89] and umbrella sampling[90]), which were originally established to study equilibrium properties, are being transferred to calculate kinetic parameters.[91] Although there are still open problems in the computational protocols, this approach seems to have a good potential to study the antibiotic-pore binding kinetics by using all-atom simulations.[71]

In another perspective approach, the molecular transport through the pore is treated as a Markov process via a network of states, the transition probabilities between which may be calculated from the all-atom MD simulations. As one may probably reasonably assume, the motion of an antibiotic along the main axis $(\mathrm{OZ})$ of a porin channel is a Brownian motion governed by the local diffusion constant $D(Z)$ and the potential of mean force, $U(Z)$. Then, the non-equilibrium probability density obeys the diffusion-drift equation, and the steady state net flux of the molecules may be calculated with the known formula:[65]

$$
J_{0}=\frac{c_{0} e^{\frac{U_{0}}{k T}}-c_{L} e^{\frac{U_{L}}{k T}}}{\int_{0}^{L} \frac{e^{\frac{U(Z)}{k T}}}{D(Z)} d Z}
$$

where, $c_{0}$ and $c_{L}$ are the concentrations of the antibiotic on each side of the channel. The potential of mean force (the free energy), which also determines the equilibrium probability density of the antibiotic inside the channel, as well as the local diffusion constant, can be calculated through allatom MD simulations by using one of the enhanced sampling methods mentioned above.

On the other hand, the required computational resources (at least two months per molecule on a single GPU) prohibit the application of these methods to the massive screening of potential new 
molecules. Therefore, a simplified and fast method to estimate the translocation rate is needed for screening virtual libraries seeking for new potential antibiotics. The simplified translocation model may be based on Eq.(A), provided that models for the free energy profile, $U(Z)$, and the effective diffusion constant, $\mathrm{D}(\mathrm{Z})$ are available. According to what we discussed in this topical review, the free energy profile may be constructed as a sum of independent contributions,

$$
U(Z)=-k T \ln \left(\frac{A(Z)}{A(0)}\right)+Q \times V(Z)-\langle\vec{E}(Z) \cdot \vec{D}\rangle+U_{\text {specific }}(Z)
$$

Here, the first term is the steric contribution due to the change of the available configuration space for the molecule, $A(Z)$, along the channel; the second term comes from the electrostatic interaction of the average potential $\mathrm{V}(\mathrm{Z})$ inside the channel and the charge of the molecule, $\mathrm{Q}$; the third term stands for the interaction of molecules dipole moment $\mathrm{D}$ with the electric field $\mathrm{E}(\mathrm{Z})$ inside the channel,[79] averaged over the possible orientations of the molecule; the last term accounts for the specific possible interactions, like H-bonds, salt bridges, hydrophobic interactions, etc; the best functional form of this last term remains to be determined. The parameters in Eq. (B) can be calculated once for each porin and each molecule, stored in a database, and then used for the screening of many molecules versus a given set of porins. A validation against the free energy evaluated with expensive all-atom simulations for few cases will help to assess the different interaction terms and apply corrections where needed.

Completion of the program of theoretical developments outlined above will provide us with the details of the translocation mechanism with the values of the quantities ready for direct experimental verification (translocation flux, kinetic parameters of molecule pore interaction, etc.).

\section{Acknowledgements}

S.A.G. is funded by EU ITN network Translocation, Nr. 607694. The research leading to these results was conducted as part of the Translocation consortium (www.translocation.com) and has received support from the Innovative Medicines Initiatives Joint Undertaking under Grant Agreement $n^{\circ} 115525$, resources which are composed of financial contribution from the European

Union's seventh framework programme (FP7/2007-2013) and EFPIA companies in kind contribution.

\section{References}

[1] WHO 2014 Antimicrobial resistance: global report on surveillance (World Health Organization) 
[2] Silver L L 2011 Challenges of antibacterial discovery Clin. Microbiol. Rev. 24 71-109

[3] Tommasi R, Brown D G, Walkup G K, Manchester J I and Miller A A 2015 ESKAPEing the labyrinth of antibacterial discovery Nat. Rev. Drug Discov. 14 529-42

[4] Lewis K 2013 Platforms for antibiotic discovery Nat. Rev. Drug Discov. 12 371-87

[5] The PEW Charitable Trusts “Antibiotics Currently in Clinical Development”

http://www.pewtrusts.org/en/multimedia/data-visualizations/2014/antibiotics-currently-in-clinicaldevelopment

[6] Laxminarayan R, Duse A, Wattal C, Zaidi A K M, Wertheim H F L, Sumpradit N, Vlieghe E, Hara G L, Gould I M, Goossens H, Greko C, So A D, Bigdeli M, Tomson G, Woodhouse W, Ombaka E, Peralta A Q, Qamar F N, Mir F, Kariuki S, Bhutta Z A, Coates A, Bergstrom R, Wright G D, Brown E D and Cars O 2013 Antibiotic resistance-the need for global solutions. Lancet Infect. Dis. 13 1057-98

[7] O’Neill J, Audi H, Knox J, Hall W, McDonnell A, Seshadri A, Mudd J, Truscott-Reid N, Toxvaerd F, Woodford N and Macdonald O 2016 Review on Antimicrobial Resistance (London: Wellcome Trust)

[8] Delcour A H 2009 Outer membrane permeability and antibiotic resistance Biochim. Biophys. Acta BBA - Proteins Proteomics 1794 808-16

[9] Fernandez L and Hancock R E W 2012 Adaptive and Mutational Resistance: Role of Porins and Efflux Pumps in Drug Resistance Clin. Microbiol. Rev. 25 661-81

[10] Nikaido H and Vaara M 1985 Molecular basis of bacterial outer membrane permeability. Microbiol. Rev. 49 1-32

[11] Benz R 1988 Structure and function of porins from gram-negative bacteria Annu. Rev. Microbiol. 42 359-93

[12] Masi M and Pagès J-M 2013 Structure, Function and Regulation of Outer Membrane Proteins Involved in Drug Transport in Enterobactericeae: the OmpF/C - TolC Case Open Microbiol. J. 7 22-33

[13] Raetz C R H and Whitfield C 2002 Lipopolysaccharide Endotoxins Annu. Rev. Biochem. 71 $635-700$

[14] Carpenter T S, Parkin J and Khalid S 2016 The Free Energy of Small Solute Permeation through the Escherichia coli Outer Membrane Has a Distinctly Asymmetric Profile J. Phys. Chem. Lett. 7 3446-51

[15] Mach T, Neves P, Spiga E, Weingart H, Winterhalter M, Ruggerone P, Ceccarelli M and Gameiro P 2008 Facilitated permeation of antibiotics across membrane channels--interaction of the quinolone moxifloxacin with the OmpF channel J. Am. Chem. Soc. 130 13301-9

[16] Mahendran K R, Hajjar E, Mach T, Lovelle M, Kumar A, Sousa I, Spiga E, Weingart H, Gameiro P, Winterhalter M and Ceccarelli M 2010 Molecular Basis of Enrofloxacin Translocation through OmpF, an Outer Membrane Channel of Escherichia coli - When Binding Does Not Imply Translocation J. Phys. Chem. B 114 5170-9

[17] Cama J, Bajaj H, Pagliara S, Maier T, Braun Y, Winterhalter M and Keyser U F 2015 Quantification of Fluoroquinolone Uptake through the Outer Membrane Channel OmpF of Escherichia coli J. Am. Chem. Soc. 137 13836-43

[18] Pagès J-M, James C E and Winterhalter M 2008 The porin and the permeating antibiotic: a selective diffusion barrier in Gram-negative bacteria Nat. Rev. Microbiol. 6 893-903

[19] Kumar A, Hajjar E, Ruggerone P and Ceccarelli M 2010 Structural and dynamical properties of the porins OmpF and OmpC: insights from molecular simulations. J. Phys. Condens. Matter 22454125

[20] Kojima S and Nikaido H 2014 High Salt Concentrations Increase Permeability through OmpC Channels of Escherichia coli J. Biol. Chem. 289 26464-73

[21] Raj Singh P, Ceccarelli M, Lovelle M, Winterhalter M and Mahendran K R 2012 Antibiotic Permeation across the OmpF Channel: Modulation of the Affinity Site in the Presence of Magnesium J. Phys. Chem. B 116 4433-8 
[22] Yoshimura F and Nikaido H 1982 Permeability of Pseudomonas aeruginosa outer membrane to hydrophilic solutes. J. Bacteriol. 152 636-42

[23] Hancock R E 1998 Resistance mechanisms in Pseudomonas aeruginosa and other nonfermentative gram-negative bacteria Clin. Infect. Dis. Off. Publ. Infect. Dis. Soc. Am. 27 Suppl 1 S93-9

[24] Hancock R E W and Brinkman F S L 2002 Function of pseudomonas porins in uptake and efflux Annu. Rev. Microbiol. 56 17-38

[25] Eren E, Vijayaraghavan J, Liu J, Cheneke B R, Touw D S, Lepore B W, Indic M, Movileanu L and van den Berg B 2012 Substrate specificity within a family of outer membrane carboxylate channels PLoS Biol. 10 e1001242

[26] Tamber S, Ochs M M and Hancock R E W 2006 Role of the novel OprD family of porins in nutrient uptake in Pseudomonas aeruginosa J. Bacteriol. 188 45-54

[27] Nikaido H 1994 Prevention of drug access to bacterial targets: permeability barriers and active efflux Science 264 382-8

[28] Blair J M A, Bavro V N, Ricci V, Modi N, Cacciotto P, Kleinekathöfer U, Ruggerone P, Vargiu A V, Baylay A J, Smith H E, Brandon Y, Galloway D and Piddock L J V 2015 AcrB drugbinding pocket substitution confers clinically relevant resistance and altered substrate specificity Proc. Natl. Acad. Sci. U. S. A. 112 3511-6

[29] Vaccaro L, Koronakis V and Sansom M S P 2006 Flexibility in a Drug Transport Accessory Protein: Molecular Dynamics Simulations of MexA Biophys. J. 91 558-64

[30] Sun J, Deng Z and Yan A 2014 Bacterial multidrug efflux pumps: mechanisms, physiology and pharmacological exploitations Biochem. Biophys. Res. Commun. 453 254-67

[31] Cowan S, Schirmer T, Rummel G, Steiert M, Ghosh R, Pauptit R, Jansonius J and Rosenbusch J 1992 Crystal-Structures Explain Functional-Properties of 2 Escherichia-Coli Porins Nature 358 727-33

[32] Baslé A, Rummel G, Storici P, Rosenbusch J P and Schirmer T 2006 Crystal Structure of Osmoporin OmpC from E. coli at 2.0 Å J. Mol. Biol. 362 933-42

[33] Tieleman D P and Berendsen H J 1998 A molecular dynamics study of the pores formed by Escherichia coli OmpF porin in a fully hydrated palmitoyloleoylphosphatidylcholine bilayer.

Biophys. J. 74 2786-801

[34] Karshikoff A, Spassov V, Cowan S W, Ladenstein R and Schirmer T 1994 Electrostatic properties of two porin channels from Escherichia coli J. Biol. Chem. 240 372-84

[35] Levadny V, Aguilella V M, Aguilella-Arzo M and Belaya M 2004 Interaction of a polar molecule with an ion channel Phys. Rev. E 70041912

[36] Nikaido H and Rosenberg E Y 1983 Porin channels in Escherichia coli: studies with liposomes reconstituted from purified proteins J. Bacteriol. 153 241-52

[37] Pratt L A, Hsing W, Gibson K E and Silhavy T J 1996 From acids to osmZ: multiple factors influence synthesis of the OmpF and OmpC porins in Escherichia coli Mol. Microbiol. 20 911-7

[38] Benz R 1994 Permeation of hydrophilic solutes through mitochondrial outer membranes: review on mitochondrial porins Biochim. Biophys. Acta 1197 167-96

[39] Kojima S and Nikaido H 2013 Permeation rates of penicillins indicate that Escherichia coli porins function principally as nonspecific channels Proc. Natl. Acad. Sci. 110 E2629-34

[40] Mahendran K R, Kreir M, Weingart H, Fertig N and Winterhalter M 2010 Permeation of Antibiotics through Escherichia coli OmpF and OmpC Porins: Screening for Influx on a SingleMolecule Level J. Biomol. Screen. 15 302-7

[41] Ferrara L G M, Wallat G D, Moynié L, Dhanasekar N N, Aliouane S, Acosta-Gutiérrez S, Pagès J-M, Bolla J-M, Winterhalter M, Ceccarelli M and Naismith J H 2016 MOMP from Campylobacter jejuni Is a Trimer of 18-Stranded $\beta$-Barrel Monomers with a Ca2 + Ion Bound at the Constriction Zone J. Mol. Biol. 428 4528-43

[42] Lou H, Chen M, Black S S, Bushell S R, Ceccarelli M, Mach T, Beis K, Low A S, Bamford V A, Booth I R, Bayley H and Naismith J H 2011 Altered Antibiotic Transport in OmpC Mutants 
Isolated from a Series of Clinical Strains of Multi-Drug Resistant E. coli ed H W van Veen PLoS ONE 6 e25825

[43] Tran Q-T, Williams S, Farid R, Erdemli G and Pearlstein R 2013 The translocation kinetics of antibiotics through porin OmpC: Insights from structure-based solvation mapping using WaterMap Proteins Struct. Funct. Bioinforma. 81 291-9

[44] Brown D G, May-Dracka T L, Gagnon M M and Tommasi R 2014 Trends and Exceptions of Physical Properties on Antibacterial Activity for Gram-Positive and Gram-Negative Pathogens $J$. Med. Chem. 57 10144-61

[45] Ziervogel B K and Roux B 2013 The Binding of Antibiotics in OmpF Porin Structure 21 $76-87$

[46] O’Shea R and Moser H E 2008 Physicochemical Properties of Antibacterial Compounds: Implications for Drug Discovery J. Med. Chem. 51 2871-8

[47] Kumar A, Hajjar E, Ruggerone P and Ceccarelli M 2010 Molecular Simulations Reveal the Mechanism and the Determinants for Ampicillin Translocation through OmpF J. Phys. Chem. B 114 9608-16

[48] Danelon C, Nestorovich E M, Winterhalter M, Ceccarelli M and Bezrukov S M 2006 Interaction of Zwitterionic Penicillins with the OmpF Channel Facilitates Their Translocation Biophys. J. 90 1617-27

[49] Hajjar E, Bessonov A, Molitor A, Kumar A, Mahendran K R, Winterhalter M, Pagès J-M, Ruggerone P and Ceccarelli M 2010 Toward Screening for Antibiotics with Enhanced Permeation Properties through Bacterial Porins Biochemistry 49 6928-35

[50] Samanta S, Scorciapino M A and Ceccarelli M 2015 Molecular basis of substrate translocation through the outer membrane channel OprD of Pseudomonas aeruginosa Phys Chem Chem Phys 17 23867-76

[51] Isabella V M, Campbell A J, Manchester J, Sylvester M, Nayar A S, Ferguson K E, Tommasi R and Miller A A 2015 Toward the Rational Design of Carbapenem Uptake in Pseudomonas aeruginosa Chem. Biol. 22 535-47

[52] Benz R, Schmid A and Vos-Scheperkeuter G H 1987 Mechanism of sugar transport through the sugar-specific LamB channel of Escherichia coli outer membrane J. Membr. Biol. 100 21-9

[53] Schwarz G, Danelon C and Winterhalter M 2003 On translocation through a membrane channel via an internal binding site: kinetics and voltage dependence Biophys. J. 84 2990-8

[54] Dutzler R, Schirmer T, Karplus M and Fischer S 2002 Translocation mechanism of long sugar chains across the maltoporin membrane channel Structure 10 1273-84

[55] Nestorovich E M, Danelon C, Winterhalter M and Bezrukov S M 2002 Designed to penetrate: time-resolved interaction of single antibiotic molecules with bacterial pores Proc. Natl. Acad. Sci. U. S. A. 99 9789-94

[56] Nestorovich E M, Rostovtseva T K and Bezrukov S M 2003 Residue ionization and ion transport through OmpF channels Biophys. J. 85 3718-29

[57] Ceccarelli M, Danelon C, Laio A and Parrinello M 2004 Microscopic Mechanism of Antibiotics Translocation through a Porin Biophys. J. 87 58-64

[58] Phale P S, Philippsen A, Widmer C, Phale V P, Rosenbusch J P and Schirmer T 2001 Role of charged residues at the OmpF porin channel constriction region probed by mutagenesis and simulation Biochemistry $\mathbf{4 0}$ 6319-25

[59] Bredin J, Saint N, Malléa M, Dé E, Molle G, Pagès J-M and Simonet V 2002 Alteration of pore properties of Escherichia coli OmpF induced by mutation of key residues in anti-loop 3 region Biochem. J. 363 521-8

[60] Malloci G, Vargiu A V, Serra G, Bosin A, Ruggerone P and Ceccarelli M 2015 A Database of Force-Field Parameters, Dynamics, and Properties of Antimicrobial Compounds Molecules 20 13997-4021

[61] Hajjar E, Mahendran K R, Kumar A, Bessonov A, Petrescu M, Weingart H, Ruggerone P, Winterhalter M and Ceccarelli M 2010 Bridging Timescales and Length Scales: From Macroscopic 
Flux to the Molecular Mechanism of Antibiotic Diffusion through Porins Biophys. J. 98 569-75

[62] Winterhalter M and Ceccarelli M 2015 Physical methods to quantify small antibiotic molecules uptake into Gram-negative bacteria Eur. J. Pharm. Biopharm. 95 63-7

[63] Schmitz K S and Schurr J M 1972 Role of orientation constraints and rotational diffusion in bimolecular solution kinetics J. Phys. Chem. 76 534-45

[64] Ceccarelli M, Vargiu A V and Ruggerone P 2012 A kinetic Monte Carlo approach to investigate antibiotic translocation through bacterial porins J. Phys. Condens. Matter 24104012 [65] Berezhkovskii A M and Bezrukov S M 2005 Optimizing Transport of Metabolites through Large Channels: Molecular Sieves with and without Binding Biophys. J. 88 L17-9

[66] Bezrukov S M, Berezhkovskii A M and Szabo A 2007 Diffusion model of solute dynamics in a membrane channel: mapping onto the two-site model and optimizing the flux J. Chem. Phys.

127115101

[67] Acosta-Gutierrez S, Scorciapino M A, Bodrenko I and Ceccarelli M 2015 Filtering with Electric Field: The Case of E. coli Porins J. Phys. Chem. Lett. 6 1807-12

[68] Scorciapino M A, D’Agostino T, Acosta-Gutierrez S, Malloci G, Bodrenko I and Ceccarelli M 2016 Exploiting the porin pathway for polar compound delivery into Gram-negative bacteria Future Med. Chem. 8 1047-62

[69] Zahn M, D’Agostino T, Eren E, Baslé A, Ceccarelli M and van den Berg B 2015 SmallMolecule Transport by CarO, an Abundant Eight-Stranded $\beta$-Barrel Outer Membrane Protein from Acinetobacter baumannii J. Mol. Biol. 427 2329-39

[70] Bodrenko I, Bajaj H, Ruggerone P, Winterhalter M and Ceccarelli M 2015 Analysis of fast channel blockage: revealing substrate binding in the microsecond range The Analyst 140 4820-7

[71] D’Agostino T, Salis S and Ceccarelli M 2016 A kinetic model for molecular diffusion through pores Biochim. Biophys. Acta BBA - Biomembr. 1858 1772-7

[72] Stavenger R A and Winterhalter M 2014 TRANSLOCATION project: how to get good drugs into bad bugs Sci. Transl. Med. 6 228ed7

[73] Wang J, Wolf R M, Caldwell J W, Kollman P A and Case D A 2004 Development and testing of a general amber force field J. Comput. Chem. 25 1157-74

[74] Doerr S, Harvey M J, Noé F and De Fabritiis G 2016 HTMD: High-Throughput Molecular Dynamics for Molecular Discovery J. Chem. Theory Comput. 12 1845-52

[75] Low A S, MacKenzie F M, Gould I M and Booth I R 2001 Protected environments allow parallel evolution of a bacterial pathogen in a patient subjected to long-term antibiotic therapy Mol. Microbiol. 42 619-30

[76] Philippsen A, Im W, Engel A, Schirmer T, Roux B and Muller D J 2002 Imaging the electrostatic potential of transmembrane channels Biophys. J. 82 1667-76

[77] Im W and Roux B 2002 Ion permeation and selectivity of OmpF porin: a theoretical study based on molecular dynamics, Brownian dynamics, and continuum electrodiffusion theory. J. Mol. Biol. 322 851-69

[78] Aguilella-Arzo M, Garcia-Celma J J, Cervera J, Alcaraz A and Aguilella V M 2007 Electrostatic properties and macroscopic electrodiffusion in OmpF porin and mutants Bioelectrochemistry 70 320-7

[79] Gutiérrez S A, Bodrenko I, Scorciapino M A and Ceccarelli M 2016 Macroscopic electric field inside water-filled biological nanopores Phys Chem Chem Phys 18 8855-64

[80] Robertson K M and Tieleman D P 2002 Orientation and interactions of dipolar molecules during transport through OmpF porin. FEBS Lett. 528 53-7

[81] Bajaj H, Scorciapino M A, Moynié L, Page M G P, Naismith J H, Ceccarelli M and Winterhalter M 2016 Molecular Basis of Filtering Carbapenems by Porins from $\beta$-Lactam-resistant Clinical Strains of Escherichia coli J. Biol. Chem. 291 2837-47

[82] Liu J, Eren E, Vijayaraghavan J, Cheneke B R, Indic M, van den Berg B and Movileanu L 2012 OccK Channels from Pseudomonas aeruginosa Exhibit Diverse Single-Channel Electrical Signatures but Conserved Anion Selectivity Biochemistry 51 2319-30 
[83] Liu J, Wolfe A J, Eren E, Vijayaraghavan J, Indic M, van den Berg B and Movileanu L 2012 Cation selectivity is a conserved feature in the OccD subfamily of Pseudomonas aeruginosa Biochim. Biophys. Acta BBA - Biomembr. 1818 2908-16

[84] Eren E, Parkin J, Adelanwa A, Cheneke B, Movileanu L, Khalid S and van den Berg B 2013 Toward Understanding the Outer Membrane Uptake of Small Molecules by Pseudomonas aeruginosa J. Biol. Chem. 288 12042-53

[85] Pothula K R and Kleinekathöfer U 2015 Theoretical analysis of ion conductance and gating transitions in the OpdK (OccK1) channel The Analyst 140 4855-64

[86] Parkin J and Khalid S 2014 Atomistic Molecular-Dynamics Simulations Enable Prediction of the Arginine Permeation Pathway through OccD1/OprD from Pseudomonas aeruginosa Biophys. J. 107 1853-61

[87] Nikaido H 2003 Molecular basis of bacterial outer membrane permeability revisited Microbiol. Mol. Biol. Rev. 67 593-656

[88] Kullman L, Winterhalter M and Bezrukov S M 2002 Transport of maltodextrins through maltoporin: a single-channel study Biophys. J. 82 803-12

[89] Laio A and Parrinello M 2002 Escaping free-energy minima Proc. Natl. Acad. Sci. U. S. A. 99 12562-6

[90] Torrie G M and Valleau J P 1974 Monte Carlo free energy estimates using non-Boltzmann sampling: Application to the sub-critical Lennard-Jones fluid Chem. Phys. Lett. 28 578-81

[91] Tiwary P and Parrinello M 2013 From Metadynamics to Dynamics Phys. Rev. Lett. 111 